\title{
Application Effect of Midwifery Nursing in the Prevention of Neonatal Asphyxia
}

\author{
Tiantian Zhu*, Nan Hao, Yan Qiao, Rongrong Ma \\ Yan'An People's Hospital, Yan'An 716000, China \\ *Corresponding author: Tian-Tian Zhu, doctor0913@163.com
}

Copyright: ( $) 2022$ Author(s). This is an open-access article distributed under the terms of the Creative Commons Attribution License (CC BY 4.0), permitting distribution and reproduction in any medium, provided the original work is cited.

\begin{abstract}
Objective: To explore the role of midwifery care in the prevention of neonatal asphyxia. Method: The 100 cases of parturient received in our hospital from January 2018 to January 2021 were divided into two groups, respectively, the control group and the experimental group with 50 cases in each group. The control group received routine care, and the experimental group received midwifery care. The psychological status of the mothers and the occurrence of neonatal asphyxia were compared between the two groups. Results: Before nursing, there was no significant difference in the mental state of the two groups of parturient $(\mathrm{P}>0.05)$. After the nursing, the mental state of the parturient in the experimental group was significantly better than that of the control group $(\mathrm{P}<0.05)$; After different ways of nursing, the incidence of asphyxia in neonates in the control group was significantly higher than that in the experimental group $(\mathrm{P}<0.05)$, and the comparison between the groups was statistically significant. Conclusion: The timely delivery of midwifery care for parturient can improve the mental state of the parturient and help reduce the incidence of neonatal asphyxia. It is worthy of clinical attention and promotion.
\end{abstract}

Keywords: Midwifery care; Parturient; Neonatal asphyxia

Online publication: January 19, 2022

\section{Introduction}

There are many reasons for neonatal asphyxia, including mothers, pregnancy-induced hypertension, anemia during pregnancy, or congenital body structure causes prolonged labor, which leads to hypoxia in newborns. Furthermore, the fetus is excessively huge, and the umbilical cord around the neck will complicate delivery. The use of midwifery forceps or fetal suction during a difficult birth can cause the fetus to have difficulties breathing, and the child's own circumstances, such as intrauterine infection, can also induce neonatal asphyxia ${ }^{[1]}$. As a result, scientific nursing approaches must be used to avoid newborn hypoxia and enhance the survival rate of neonates ${ }^{[2-3]}$. This study took 100 cases of parturient as the research object, and the main purpose was to analyze the impact of the use of midwifery care on the prevention of neonatal asphyxia:

\section{Materials and methods}

\subsection{Basic information}

We selected 100 women who were admitted to our hospital between January 2018 and January 2021 as the research objects, and were divided into two groups using a random number table method. The control group was 21-37 years old, with an average of 28.56 3 3.31 years old. The experimental group was 22-36 years old, with an average of $27.01 \pm 2.83$ years old. There was no significant difference in the general information of the two groups of parturient, not statistically significant, and comparable $(\mathrm{p}>0.5)$. 


\subsubsection{Inclusion criteria}

No cognitive dysfunction, no mental retardation, able to carry out effective daily communication. Family members and mothers themselves all participate in the experiment voluntarily and sign an informed consent form.

\subsubsection{Exclusion criteria}

Suffering from mental disorders, severe coagulopathy, drug allergies, unable to operate normally, severe organ dysfunction.

\subsection{Methods}

\subsubsection{The control group used conventional nursing methods}

2.2.2. The experimental group adds midwifery nursing methods on the basis of routine nursing as follows

(1) Before performing midwifery care, the nursing staff should fully understand the specific information of the parturient, and instruct the parturient to perform routine prenatal examinations, record the vital indicators of the parturient such as heart rate, blood pressure, pulse, etc. to determine the basic situation. Nursing staff can understand the fetal heart rate and position of the fetus through the results of ultrasound diagnosis, and observe the specific conditions of the fetus in the womb. They can explain the specific process of delivery, possible accidents, and the main precautions after the end, so that the mother can breathe correctly, and avoid fetal distress during the delivery process.

(2) Prepare in advance the medical equipment and drugs that may be needed during the delivery process. During the delivery process, the midwife nurse can squeeze the mucus and amniotic fluid in the fetus's nose and mouth. Before the fetus breathes for the first time, it needs to be cleaned by suction, and the newborn's head can be slightly lifted. After cleaning, massage his limbs and back, and then inhale oxygen until the newborn's skin turns red. In the case of neonatal asphyxia, airbags should be used immediately for oxygen supply, and artificial respirators should be used if necessary.

(3) Post-natal care: After delivery, midwifery nurses should closely monitor the specific conditions and vital signs of some maternal newborns, and observe whether they need to be treated with drugs according to their actual conditions.

\subsection{Observation indicators}

2.3.1. Compare the mental state of the two groups of mothers, including depression score and anxiety score

2.3.2. Compare the occurrence of asphyxia in the two groups of newborns

\subsection{Statistical method}

The SPSS17.0 system was used to process the two sets of maternal data, and the obtained data were subjected to t-test, expressed in $\mathrm{x} \pm \mathrm{s}$, and count data were subjected to $\mathrm{X}^{2}$ test, expressed in $\%$. It is statistically significant when $\mathrm{P}<0.05$.

\section{Result}

\subsection{Compare the SAS and SDS scores of the two groups of patients before and after nursing}

Before nursing, there was no significant difference in the two scores of the mental state of the two groups of women ( $\mathrm{P}>0.05)$. After nursing, the negative mental state of the women in the experimental group and the control group was significantly improved $(\mathrm{P}<0.05)$. Compared with the status scores, the two scores of the pregnant women in the experimental group were lower than those in the control group $(\mathrm{P}<0.05)$, which 
was statistically significant, as shown in Table 1.

Table 1. Comparison of SAS and SDS scores between the two groups before and after nursing ( $\mathrm{x} \pm \mathrm{s})$

\begin{tabular}{ccccc}
\hline Group & SAS (points) & \multicolumn{2}{c}{ SDS (points) } \\
\hline & Before care & After care & Before care & After care \\
\hline Experimental group $(\mathbf{n = 5 0})$ & $53.52 \pm 3.26$ & $41.67 \pm 2.57$ & $56.45 \pm 1.90$ & $42.76 \pm 1.45$ \\
Control group $(\mathbf{n = 5 0}$ & $53.44 \pm 3.58$ & $47.42 \pm 3.18$ & $56.45 \pm 1.52$ & $48.67 \pm 1.53$ \\
$\mathbf{t}$ & 0.1168 & 9.9442 & 0.0000 & 19.8251 \\
$\mathbf{P}$ & 0.9072 & 0.0000 & 1.0000 & 0.0000 \\
\hline
\end{tabular}

\subsection{Comparison of suffocation}

The incidence of neonatal asphyxia in the experimental group was significantly lower than that in the control group $(\mathrm{P}<0.05)$, and the comparison was statistically significant, as shown in Table 2.

Table 2. Comparison of the occurrence of suffocation (n, \%)

\begin{tabular}{cccccc}
\hline Group & Number of cases & $\begin{array}{c}\text { Severe } \\
\text { suffocation }\end{array}$ & $\begin{array}{c}\text { Mild } \\
\text { suffocation }\end{array}$ & $\begin{array}{c}\text { No } \\
\text { suffocation }\end{array}$ & Suffocation \\
\hline Experimental group & 50 & $0(0.00)$ & $(0.00)$ & $50(100.00)$ & $0(0.00)$ \\
Control group & 50 & $1(2.00)$ & $4(8.00)$ & $45(90.00)$ & $5(10.00)$ \\
$\mathbf{X}^{\mathbf{2}}$ & & & & & 5.2632 \\
$\mathbf{P}$ & & & & & 0.0218 \\
\hline
\end{tabular}

\subsection{Discussion}

During pregnancy and childbirth, women have relatively higher risks. The main reasons are that they are under greater psychological pressure and are very worried about the safety of themselves and the fetus. In addition, mothers are prone to multiple complications and serious negative emotions ${ }^{[4-5]}$. In recent years, with the continuous enhancement of our national strength, the medical industry has developed rapidly, and people have paid more and more attention to women during pregnancy. Midwifery nursing is a new clinical nursing model that can break the limitations of traditional nursing. The mothers are given scientific care throughout the birth process, thereby increasing their natural delivery rate and effectively preventing the occurrence of neonatal asphyxia ${ }^{[6]}$. In this study, before nursing, there was no significant difference between the two groups of patients' mental state scores $(\mathrm{P}>0.05)$. After nursing, the negative mental state of the experimental group and the control group were significantly improved $(\mathrm{P}<0.05)$. The mental state scores between the two groups were compared. The two scores of the experimental group were lower than those of the control group $(\mathrm{P}<0.05)$, which was statistically significant; the incidence of neonatal asphyxia in the experimental group was significantly lower than that in the control group $(\mathrm{P}<0.05)$, which fully demonstrates that this scientific nursing method can improve the mental state of mothers and reduce the incidence of neonatal asphyxia.

In summary, in maternal nursing, the application of midwifery nursing can improve the mental state of the maternal. Therefore, this nursing method is worthy of promotion in clinical practice in the future.

\section{Disclosure statement}

The author declares no conflict of interest. 


\section{References}

[1] Zhang L, Wang W, Gong K, 2021, The Effect of Structured Midwifery Nursing Combined with Lamaze Training on Delivery Methods and Delivery Outcome of Parturients. Henan Medical Research, 30(31): 5916-5919.

[2] Zeng Y, Cao J, 2021, The Effect of Pregnancy Weight Management Combined with Continuous Midwifery Care Model on Reducing Pregnancy Complications, Dystocia and Cesarean Section Rate of Primipara. Guizhou Medicine, 45(10): 1647-1648.

[3] Li S, 2021, Study on the Application of Continuous Quality Improvement and Joint Responsibility Midwifery in the Nursing of the Delivery Room. Chinese Community Physician, 37(30): 160-161.

[4] Deng F, 2021, Effects of Doula Midwifery Nursing Care on the Outcome and Psychological State of Delivery and Pregnancy of Term Pregnancy and First Parturients. Smart Health, 7(27): 82-84. DOI: 10.19335/j.cnki.2096-1219.2021.27.028

[5] Zhang W, Li Z, Yang C, et al., 2021, The Effect of Unprotected Midwifery Nursing in Promoting the Natural Delivery of Primiparas and the Analysis of the Impact of Perineal Pain VAS Score. Systems Medicine, 6(15): 128-131. DOI: 10.19368/j.cnki.2096-1782.2021.15.128

[6] Liang H, 2021, The Influence of Kolb Experiential Teaching on the Humanistic Care Level and Core Competence of Obstetric Midwives. Nursing Practice and Research, 18(14): 2181-2184.

Publisher's note

Bio-Byword Scientific Publishing remains neutral with regard to jurisdictional claims in published maps and institutional affiliations. 